\title{
Forced oscillations with continuum models of Atomic force microscopy
}

\author{
Julio R. Claeyssen*, Teresa Tsukazan*, Leticia Tonetto* and Jose M. Balthazar ${ }^{\dagger}$ \\ *Institute of Mathematics, Universidade Federal do Rio Grande do Sul, Porto Alegre,Brazil \\ ${ }^{\dagger}$ Department of Applied Mathematics, Universidade Estadual de Sao Paulo, Rio Claro, Brazil
}

\begin{abstract}
The dynamics of the AFM-atomic force microscope follows a model based in a Timoshenko cantilever beam with a tip attached at the free end and acting with the surface of a sample. General boundary conditions arise when the tip is either in contact or non-contact with the surface. The governing equations are given in matrix conservative form subject to localized loads. The eigenanalysis is done with a fundamental matrix response of a damped second-order matrix differential equation. Forced responses are found by using a Galerkin approximation of the matrix impulse response. Simulations results with harmonic and pulse forcing show the filtering character and the effects of the tip-sample interaction at the end of the beam.
\end{abstract}

Keywords: Atomic force microscopy, Timoshenko beam, impulse matrix response, forced responses, Galerkin method PACS: 68.37.Ps, 46.70.De, *43.20.Tb, 02.70.Dh, 02.70.Hm, 42.30.Lr, 78.67.Sc

\section{INTRODUCTION}

Atomic force microscopy (AFM) is a scanning probe microscopy (SPM) technique [1] to obtain images of surface topography at the atomic scale, in a noninvasive manner, from a wide variety of samples on a scale from angstroms to 100 microns. A typical AFM consists of a micro-cantilever with a sharp tip, a sample positioning system, a detection system and a control system (Fig. 1). In terms of the cantilever state of motion during measurement, the two basic types of AFM modes are: static mode (contact, friction or lateral force) and dynamic mode (noncontact, tapping or semi-contact, acoustic, piezo, electrostatic,etc) [2].

With the versatility of use of the AFM, such as nanomachining or as a platform for chemical and biological sensors in connection with and surface and thermal effects, the effects of transverse shear deformation and rotary inertia on the frequency are significant [3]. With smaller values of the ratio of the probe length to its thickness, the Timoshenko beam theory is able to predict the frequencies of flexural vibrations of the higher modes with higher stiffness for the AFM cantilevers [4]. In this paper, the AFM is mathematically modeled as a Timoshenko microcantilever with a sharp tip located at the free end and subject to a distributed time-dependent external forcing and boundary conditions at the end of the beam $[5,6,7]$. For studying the Timoshenko beam subject to a variety of tip-interaction situation, we shall introduce a matrix formulation that will help to describe basic dynamic modes of AFM in a unified manner.

Our findings are given in terms of the initial-value Green matrix response or distributed impulse response.

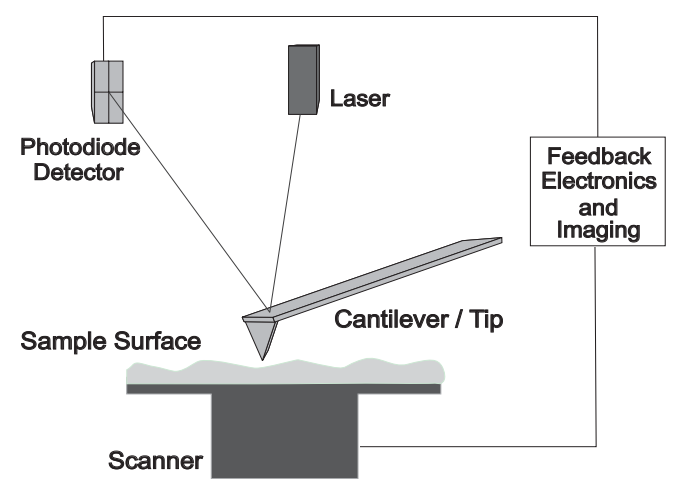

FIGURE 1. Schematic of an Atomic Force Microscope operation

This allows to characterize transients and forced responses of quite general dynamic AFM operational modes. The vibration modes for general tip-sample interaction are explicitly given in terms of a fundamental matrix response of a second-order matrix modal differential equation. This response can be determined in closed form $[8,9]$ and it is observed that has a completely oscillatory behaviour beyond a critical frequency value. This somehow reflects the hyperbolicity of the Timoshenko model in contrast to the parabolic type Euler-Bernoulli model.

Galerkin method is used in matrix form to approximate the matrix distributed impulse response from a concentrated matrix response. Forced responses are determined by convolution with a concentrated impulse response and a localized response at the end of the beam due to boundary conditions. Simulations results with 
piecewise forcing or harmonic driving forces show the filtering character of the model when considering highfrequencies and output shapes. It is also compared the effects of the tip-sample interaction with those of a microcantilever beam. This methodology could be also employed in the case of composite beams.

\section{FLEXURAL VIBRATIONS OF AFM USING THE TIMOSHENKO MODEL}

Although AFM was developed for producing highresolution images of surface structures, it has been recently used as a nanodevice cutting technique. This leads to use microbeams that are more rigid than those in conventional AFM cantilevers for measurements. Thick beams have relatively high transverse shear modulus and the effects of rotary inertia and transverse shear deformation must be used in the dynamic analysis of such beams. The Timoshenko model corrects the classical beam theory with first-order shear deformation effects. This model rest on the assumptions of small deformations and linear elastic isotropic material behavior. We consider the micro-cantilever having length $L$, uniform cross section $A$ and mass density area of the beam $\rho$. We let $I$ be the moment of inertia of the cross section area, $w(t, x)$ the flexural deflection of the beam, $\psi(t, x)$ the rotation angle of cross section of the beam, $f(t, x)$ a transverse dynamic load and $q(t, x)$ and moment load. The governing equations are given by [10]

$$
\begin{aligned}
& \rho A w_{t t}-\kappa G A\left(w_{x x}-\psi_{x}\right)=f(t, x) \\
& \rho I \psi_{t t}-E I \psi_{x x}-\kappa G A\left(w_{x}-\psi\right)=q(t, x)
\end{aligned}
$$

The boundary conditions are those of a cantilever beam or subject to balance of the moment and shear at the free end $x=L$.

\section{Matrix formulation}

The coupled Timoshenko model Eqn. (1) can be written as a second-order differential equation with matrix coefficients

$$
M \ddot{\mathbf{v}}+\mathrm{K} \mathbf{v}=\mathrm{F}
$$

where

$$
\begin{gathered}
\mathrm{v}=\left(\begin{array}{l}
w(t, x) \\
\phi(t, x)
\end{array}\right), \mathrm{F}=\left(\begin{array}{c}
f(t, x) \\
q(t, x)
\end{array}\right) \\
\mathrm{M}=\left(\begin{array}{cc}
\rho A & 0 \\
0 & \rho I
\end{array}\right), \quad \mathrm{K}=\mathrm{E} \frac{\partial^{2}}{\partial x^{2}}+\mathrm{N} \frac{\partial}{\partial x}+\mathrm{R}
\end{gathered}
$$

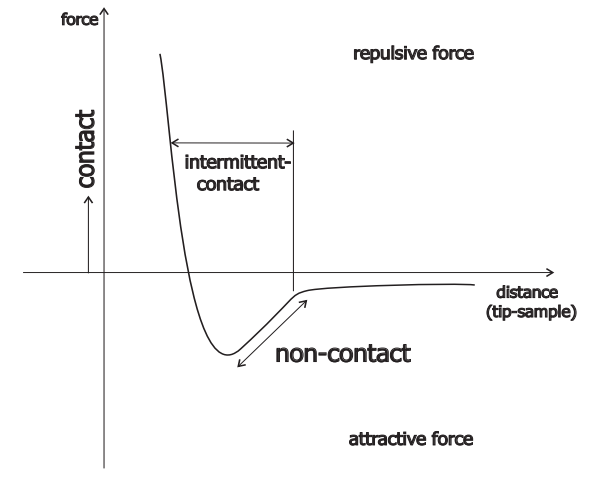

FIGURE 2. Van der Waals force-tip-sample curve

with

$$
\begin{aligned}
& \mathrm{E}=\left(\begin{array}{cc}
-\kappa G A & 0 \\
0 & -E I
\end{array}\right), \mathrm{N}=\left(\begin{array}{cc}
0 & 0 \\
0 & \kappa G A
\end{array}\right), \\
& \mathrm{R}=\left(\begin{array}{cc}
0 & 0 \\
0 & -\kappa G A
\end{array}\right) .
\end{aligned}
$$

It is frequently found in the literature, that the unforced Timoshenko model is decoupled into the same fourth-order time differential equation for the deflection and rotating angle [11]. However,the boundary conditions actually couple the system. Only for very few cases the unforced linear case is completely decoupled. For the case of a forced Timoshenko model, the transverse forcing and moment load has to be regular in order to admit differentation. These arguments suggest the convenience of keeping the original second-order physical formulation.

\section{THE DYNAMIC RESPONSE}

The total dynamic deflection of the AFM microbeam towards the sample and rotation angle about its equilibrium can be written as

$$
\begin{aligned}
& w(t, x)=u(t, x)+w^{*}(x)+y(t) \\
& \psi(t, x)=\phi(t, x)+\phi^{*}(x)
\end{aligned}
$$

where $u(t, x), \psi(t, x)$ denote the deflection and rotation relative to a non-inertial frame attached to the moving base $y(t)$, $w^{*}(x), \phi^{*}(x)$ the configuration shall not consider the length of tip as in [6]. Thus

$$
\mathrm{v}=\mathrm{u}+\mathrm{v}^{*}+\mathrm{a}(t)
$$

where

$$
\mathrm{u}=\left(\begin{array}{c}
u(t, x) \\
\phi(t, x)
\end{array}\right), \mathrm{a}(t)=\left(\begin{array}{c}
y(t) \\
0
\end{array}\right) .
$$


and

$$
\mathrm{u}^{*}=\left(\begin{array}{c}
u^{*}(x) \\
\phi^{*}(x)
\end{array}\right),
$$

being the steady equilibrium $\mathrm{Ku}^{*}=\mathrm{F}$.

For a uniform beam, the stiffness operator in Eqn. (9) is time independent. By substituting $v=u+v^{*}+a$, it turns out

$$
\mathrm{Mü}+\mathrm{Ku}=\mathrm{f}
$$

where we have the forcing term $f=F-\left(M \ddot{a}+K v^{*}\right)$.

\section{AFM-Tip-cantilever interactions}

When the tip processes a sample surface in contact mode, the AFM cantilever moves down vertically with small amplitude. For AFM in noncontact mode, the interaction between the AFM tip and the surface considers conservative forces such as those derived form the Leonard-Jones potential [12] and the tip remains above certain distance from the sample surface avoiding eventual destruction of the sample surface [13]. In the semi-contact mode or tapping mode, the there are nonlinear forces that include the attractive van der Waals forces and the short-range interatomic repulsive interactions and contact nonlinearities (Fig. 2) [7].

The tip interaction with the sample has been usually modeled as being subject to springs or dash-springs for normal and lateral interaction and to an external excitation of the base $[14,15,16]$. For instance, when the tip of length $h$ and mass $m$ is subject to normal $k_{N}$ and lateral springs $k_{L}$ and viscous dampers $c_{N}, c_{L}$, the moment and shear conditions at the free end are given by [4]

$$
\begin{array}{r}
E I \frac{\partial \phi}{\partial x}=-k_{L} h^{2} \phi-c_{L} h^{2} \frac{\partial \phi}{\partial t}-\left.m c^{2} \frac{\partial^{2} \phi}{\partial t^{2}}\right|_{x=L} \\
\kappa G A\left(\frac{\partial w}{\partial x}-\phi\right)=-k_{N} w(t, L)-c_{N} \frac{\partial w}{\partial t}-\left.m \frac{\partial^{2} w}{\partial t^{2}}\right|_{x=L}
\end{array}
$$

where $c$ denotes the distance between the lower edge of the cantilever and the centroid of the cross section.

More complex descriptions of the tip-sample force include non-linear surface and contact forces at the boundary $x=L$ due to Derjaguin-Muller-Toporev (DMT), Johnson-KendallRoberts (JKT) [17], [18]. Lateral interactions are also to be considered when the AFM acts as nano-manipulation tool for direct mechanical nanomachining, indentation and cutting of solid materials (Fig. 3) [19],[20].

In the literature, the tip-surface interaction $F_{t s}$ is usually treated as a boundary condition or a localized excitation [6],[21],[22]. The case of jump discontinuities can be treated with generalized functions [23]. In noncontact atomic force microscopy, the effects of a contribution are of the type

$$
\mathrm{F}_{\mathrm{ts}}=f(t) \delta(x-L)
$$

where $f(t)$ is the localized tip-surface interaction, while for a semi-contact mode it is written as

$$
\mathrm{F}_{\mathrm{ts}}=R\left(Z-u(t, L)-w_{*}(L)-y(t)\right) \delta(x-L)
$$

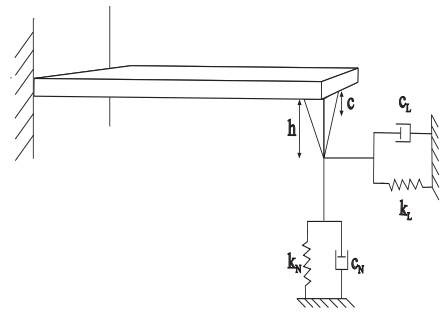

FIGURE 3. AFM tip-sample interaction

where $Z$ is the gap between the sample and probe tip in the reference configuration.

\section{A unified dynamic AFM model}

The basic AFM operation modes discussed above (contact, non-contact and semi-contact), can be encompassed within the matrix Timoshenko model

$$
\begin{aligned}
& \mathrm{M} \ddot{v}(t, x)+ \mathrm{Kv}(t, x)=\mathrm{F}, 0<x, \xi<L, t>0 \\
& \mathrm{v}(0, x)=\mathrm{v}_{o}(x), \quad \mathrm{v}_{t}(0, x)=\mathrm{v}_{1}(x) \\
& \\
& \operatorname{Av}(t, 0)+\mathrm{Jv}_{x}(t, 0)=\mathrm{f}_{1} \\
& \mathrm{Fv}(t, L)+\mathrm{Qv}_{x}(t, L)=\mathrm{f}_{2}
\end{aligned}
$$

where $F$ can include driven excitations or hydrodynamic damping and $f_{1}, f_{2}$ interactions terms with the free end. For instance,

$$
\begin{gathered}
\mathrm{f}_{1}=-\mathrm{Cv}(t, 0)-\mathrm{Dv}_{t}(t, 0)-\mathrm{Ev}_{t t}(t, 0) \\
\mathrm{f}_{2}=-\mathrm{Gv}(t, L)-\mathrm{Pv}_{t}(t, L)-\mathrm{Sv}_{t t}(t, L) .
\end{gathered}
$$

In Eqn. (10), the given conditions at $x=L$ will have

$$
\begin{gathered}
\mathrm{G}=-\left(\begin{array}{cc}
k_{N} & -\kappa G A \\
0 & k_{L} h^{2}
\end{array}\right), \mathrm{P}=-\left(\begin{array}{cc}
c_{N} & 0 \\
0 & c_{L} h^{2}
\end{array}\right) \\
\mathrm{S}=-\left(\begin{array}{cc}
m & 0 \\
0 & m c^{2}
\end{array}\right)
\end{gathered}
$$

We point out that although the unforced governing equations are given in conservative form, the boundary conditions might change such character.

When using the classical modal analysis of a cantilever beam, the boundary conditions become

$$
\left(\begin{array}{ll}
1 & 0 \\
0 & 1
\end{array}\right) \mathrm{v}(t, 0)+\left(\begin{array}{ll}
0 & 0 \\
0 & 0
\end{array}\right) \mathrm{v}_{x}(t, 0)=0
$$

$\left(\begin{array}{rr}0 & 0 \\ 0 & -1\end{array}\right) \mathrm{v}(t, 0)+\left(\begin{array}{ll}0 & 1 \\ 1 & 0\end{array}\right) \mathrm{v}_{x}(t, L)=0$ 
and interaction boundary conditions can be considered as localized forces at the free end. In this situation, non-classical modal analysis can be performed in terms of cantilever modes.

\section{The matrix impulse response}

The dynamic response of the Timoshenko model Eqn. (1) or Eqn.(9) can be described in terms of the matrix impulse response or matrix Green function $\mathbf{h}(t, x, \xi)$ of the associated homogeneous initial-value problem (13) and it has been obtained in $[24]$ as

$$
\begin{aligned}
& \mathrm{v}(t, x)=\int_{0}^{t} \int_{0}^{L}\left(\mathbf{h}_{t}(\tau, x, \xi) \mathrm{Mv}_{o}(\xi)+\mathbf{h}(\tau, x, \xi) \mathrm{Mv}_{1}(\xi)\right) d \xi d \tau \\
& \quad+\int_{0}^{t} \int_{0}^{L} \mathbf{h}(t-\tau, x, \xi) \mathrm{f}(\tau, \xi) d \xi d \tau+\left.\mathrm{J}(\mathrm{v}, \mathbf{h})\right|_{0} ^{L}
\end{aligned}
$$

where $\mathrm{J}$ is a term containing effects of the initial-value Green function with values of $v$ at the boundary. In particular, the boundary term for a cantilever beam with a time dependent boundary condition $f_{2}(t)$ at the free end has been identified as

$$
J=\int_{0}^{t} \mathbf{h}(t-\tau, x, L) \mathrm{E}^{T} \mathrm{Qf}_{2}(\tau) d \tau
$$

where

$$
\mathrm{E}=\left(\begin{array}{cc}
-\kappa G A & 0 \\
0 & -E I
\end{array}\right), \quad \mathrm{Q}=\left(\begin{array}{cc}
0 & 1 \\
1 & 0
\end{array}\right)
$$

We can observe that the forced response given by Eqn. (17) will involve the convolution of the impulse response and distributed or concentrated forcing effects as in (18).

\section{Frequency response}

In practice, when computing the convolution integral for the forced response, we actually have

$$
\mathrm{v}(t, x)=\mathrm{v}_{h}(t, x)+\mathrm{v}_{p}(t, x)
$$

where $\mathrm{v}_{h}(t, x)$ is a free vibration introduced by the system and whose initial values are a priori unknown. It turns out that these initial values are supplied by the permanent response $\mathrm{v}_{p}(t, x)$ that can be determined by other means.

Since the impulse response and its time derivative constitute a basis for the free responses and the forced response Eqn. (17) has null initial values at $t=0$, the induced system free response due to a permanent response $\mathrm{v}_{p}(t, x)$ can be easily determined. It turns out

$$
\begin{aligned}
\mathrm{v}_{h}(t, x)= & -\int_{0}^{L} \mathbf{h}_{1}(t, x, \xi) \dot{\mathrm{v}}_{p}(0, \xi) d \xi \\
& -\int_{0}^{L} \mathbf{h}_{o}(t, x, \xi) \mathrm{v}_{p}(0, \xi) d \xi
\end{aligned}
$$

where

$$
\begin{array}{r}
\mathbf{h}_{1}(t, x, \xi) \phi(\xi)=\mathbf{h}(t, x, \xi) \mathrm{M} \phi(\xi) \\
\mathbf{h}_{o}(t, x, \xi) \phi(\xi)=\frac{\partial \mathbf{h}_{t}(t, x, \xi)}{\partial t} \mathrm{M} \phi(\xi)
\end{array}
$$

Harmonic and piecewise linear forcing are of interest in frequency analysis. When seeking a response of the same type the transfer function is introduced. Given the harmonic input

$$
f(t, x)=e^{i \omega t} \mathrm{v}(x)
$$

we have the harmonic output response

$$
\mathrm{v}_{p}(t, x)=e^{i \omega t} \mathbf{H}(i \omega) \mathrm{v}(x)
$$

where

$$
\mathbf{H}(i \omega) v(x)=\int_{0}^{L} \mathbf{H}(i \omega, x, \xi) \mathrm{v}(\xi) d \xi .
$$

The kernel $\mathbf{H}(s, x, \xi)$ of the transfer operator $\mathbf{H}$ is the Laplace transform of the impulse response $\mathbf{h}(t, x, \xi)$. In particular, for a concentrated force at a point $x=a$ of spatial amplitude

$$
\mathrm{v}(x)=v(x) \delta(x-a)
$$

we have the permanent response

$$
\mathrm{v}_{p}(t, x)=e^{i \omega t} \mathbf{H}(i \omega, x, a) v(a)
$$

With the initial values $\mathrm{v}_{p}(0, \xi)=\mathbf{H}(i \omega, \xi, a) v(a), \dot{\mathrm{v}}_{p}(0, \xi)=$ $i \omega v_{p}(0, \xi)$, the induced free response is given by

$$
\mathrm{v}_{h}(t, x)=-\int_{0}^{L} \mathrm{r}(t, x, \xi, \omega) \operatorname{MH}(i \omega, a, \xi) v(a) d \xi
$$

where

$$
\mathrm{r}=\mathbf{h}_{t}(t, x, \xi)+i \omega \mathbf{h}(t, x, \xi)
$$

For a pulse amplitude

$$
\mathrm{v}(x)=\mathrm{v}_{o}(\text { Heavisde }(x-L+b)-\text { Heaviside }(x-L))
$$

the permanent response turns out

$$
\mathrm{v}_{p}(x)=e^{i \omega t} \int_{L-b}^{L} H(i \omega, x, \xi) \mathrm{v}_{o} d \xi .
$$

As before, by substituting the initial values in (20), the induced free response will now be

$$
\mathrm{v}_{h}(t, x)=-\int_{0}^{L} \mathrm{r}(t, x, \xi, \omega) \operatorname{MH}(i \omega, 0, \xi) \mathrm{v}(\xi) d \xi
$$

with $r$ given as in (28). In the case of a time linear exponential forcing

$$
f(t, x)=\exp (\lambda t)(c t+d)
$$

we have the particular solution

$$
w p(t, x)=\exp (\lambda t)(t C+D)
$$

where

$$
\begin{aligned}
C & =\left(\lambda^{2} M+K\right)^{-1} c \\
D & =\left(\lambda^{2} M+K\right)^{-1} d-2 \lambda\left(\lambda^{2} M+K\right)^{-2} M c
\end{aligned}
$$

whenever $\lambda$ is not an eigenvalue or natural frequency. 


\section{FREE TRANSVERSE VIBRATIONS}

The search of exponential solutions

$$
\mathrm{v}(t, x)=e^{\lambda t} \mathbf{v}(x), \quad \mathbf{v}(x)=\left(\begin{array}{c}
w(x) \\
\psi(x)
\end{array}\right)
$$

of the unforced linearized system underlying the Timoshenko model

$$
\mathrm{M} \frac{\partial^{2} \mathrm{v}}{\partial t^{2}}+\mathrm{Kv}=0
$$

subject to linear general separated homogeneous boundary conditions

$$
\begin{aligned}
& a_{11} w(t, 0)+a_{12} \phi(t, 0)+j_{11} w_{x}(t, 0)+j_{12} \phi_{x}(t, 0)=L B_{11} \\
& a_{21} w(t, 0)+a_{22} \phi(t, 0)+j_{21} w_{x}(t, 0)+j_{22} \phi_{x}(t, 0)=L B_{12} \\
& f_{11} w(t, L)+f_{12} \phi(t, L)+q_{11} w_{x}(t, L)+q_{12} \phi_{x}(t, L)=L B_{21} \\
& f_{21} w(t, L)+f_{22} \phi(t, L)+q_{21} w_{x}(t, L)+q_{22} \phi_{x}(t, L)=L B_{22}
\end{aligned}
$$

where $L B$ denotes linear boundary conditions involving $w, \dot{w}$ and $\ddot{w}$ at $x=L$ or in matrix terms

$$
\begin{aligned}
\mathrm{Av}(t, 0)+\mathrm{Jv}_{x}(t, 0) & =\mathrm{LB}_{1} \\
\mathrm{Fv}(t, L)+\mathrm{Qv}_{x}(t, L) & =\mathrm{LB}_{2} .
\end{aligned}
$$

leads to a quadratic eigenvalue problem. It amounts to determine nontrivial solutions of the second-order differential equation with matrix coefficients

$$
\mathbf{A v}^{\prime \prime}(\mathbf{x})+\mathbf{B} \mathbf{v}^{\prime}(\mathbf{x})+\mathbf{C}(\lambda) \mathbf{v}(\mathbf{x})=\mathbf{0},
$$

that satisfy the boundary conditions

$$
\begin{aligned}
& \mathrm{A} \mathbf{v}(0)+\mathrm{J} \mathbf{v}^{\prime}(0)=\mathrm{LB}_{1} \\
& \mathrm{~F} \mathbf{v}(L)+\mathrm{Q} \mathbf{v}^{\prime}(L)=\mathrm{LB}_{2}
\end{aligned}
$$

where $A, J, F$ and $Q$ are $2 \times 2$ matrices and the $L^{\prime}$ s are $2 \times 1$. The matrix coefficients of the second-order differential system Eqn. (38) are

$$
\begin{aligned}
& \mathbf{A}=\left(\begin{array}{cc}
-\kappa G A & 0 \\
0 & -E I
\end{array}\right), \mathbf{B}=\left(\begin{array}{cc}
0 & \kappa G A \\
-\kappa G A & 0
\end{array}\right), \\
& \mathbf{C}(\lambda)=\left(\begin{array}{rr}
\rho A \lambda^{2} & 0 \\
0 & \lambda^{2} E I+\kappa G A
\end{array}\right) .
\end{aligned}
$$

We should observe that if viscous damping forces are considered, then the matrix $\mathbf{M}$ has to be modify to include an eigenvalue term. Also, when considering localized linearized tip-sample interactions and viscous damping force acting on a microcantilever beam (clamped-free), the eigenvalue problem will modify the coefficient matrices in Eqn. (35) but the boundary conditions Eqn. (37) will be those of clamped-free beam.

For the situation of composite multispan beams, we have continuity an equilibrium conditions at the junctions where there are discontinuities due to different material or acting devices. In general, assuming $N$ spans, at the $j-t h$ junction we have to fullfill a set of compatibility conditions $\left.\mathscr{C}_{1, j} \mathbf{w}_{j}\left(t, x_{j}\right)=\mathscr{C}_{2, j} \mathbf{w}_{j+1}\left(t, x_{j}\right)\right)$, where $\mathrm{w}_{j}(t, x)=\left(w_{j}(t, x), \phi_{j}(t, x), w_{j}^{\prime}(t, x), \phi_{j}^{\prime}(t, x)\right)^{T}, \quad \mathrm{j}=1: \mathrm{N}-1$, with the matrices $\mathscr{C}_{1, j}, \mathscr{C}_{2, j}$, involving the coefficients in the continuity and equilibrium conditions

$$
\begin{aligned}
& E_{11}^{(j)} w_{j}\left(t, x_{j}\right)+F_{11}^{(j)} \phi_{j}\left(t, x_{i}\right)+G_{11}^{(j)} w_{i}^{\prime}\left(t, x_{j}\right)+H_{11}^{(j)} \phi_{j}^{\prime}\left(t, x_{j}\right) \\
&=E_{12}^{(j)} w_{j+1}\left(x_{j}, t\right)+F_{12}^{(j)} \phi_{j+1}\left(t, x_{j}\right)+G_{12}^{(j)} w_{j+1}^{\prime}\left(t, x_{j}\right) \\
& H_{12}^{(j)} \phi_{j+1}^{\prime}\left(t, x_{j}\right) \\
& E_{21}^{(j)} w_{j}\left(t, x_{j}, t\right)+F_{21}^{(j)} \phi_{j}\left(t, x_{i}\right)+G_{21}^{(j)} w_{j}^{\prime}\left(t, x_{j}\right)+H_{21}^{(j)} \phi_{j}^{\prime}\left(t, x_{j}\right) \\
& \quad=E_{22}^{(j)} w_{j+1}\left(x_{j}, t\right)+F_{22}^{(j)} \phi_{j+1}\left(t, x_{j}\right)+G_{22}^{(j)} w_{j+1}^{\prime}\left(x_{j}, t\right) \\
& \quad+H_{22}^{(j)} \phi_{j+1}^{\prime}\left(t, x_{j}\right) \\
& E_{31}^{(j)} w_{j}\left(x_{j}, t\right)+F_{31}^{(j)} \phi_{j}\left(t, x_{j}\right)+G_{31}^{(j)} w_{j}^{\prime}\left(x_{j}, t\right)+H_{31}^{(j)} \phi_{j}^{\prime}\left(t, x_{j}\right) \\
& \quad=E_{32}^{(j)} w_{j+1}\left(x_{j}, t\right)+F_{32}^{(j)} \phi_{j+1}\left(t, x_{j}\right)+G_{32}^{(j)} w_{j+1}^{\prime}\left(t, x_{j}\right) \\
& \quad+H_{32}^{(j)} \phi_{j+1}^{\prime}\left(t, x_{j}\right) \\
& E_{41}^{(j)} w_{j}\left(x_{j}, t\right)+F_{41}^{(j)} \phi_{j}\left(t, x_{j}\right)+G_{41}^{(j)} w_{j}^{\prime}\left(t, x_{j}\right)+H_{41}^{(j)} \phi_{j}^{\prime}\left(t, x_{j}\right) \\
& \quad=E_{42}^{(j)} w_{j+1}\left(x_{j}, t\right)+F_{42}^{(j)} \phi_{j+1}\left(t, x_{j}\right)+G_{42}^{(j)} w_{j+1}^{\prime}\left(t, x_{j}\right) \\
& \quad+H_{42}^{(j)} \phi_{j+1}^{\prime}\left(t, x_{j}\right)
\end{aligned}
$$

\section{Modal analysis}

The general solution of the second-order matrix differential equations Eqn. (38) in terms of initial values is given by

$$
\begin{gathered}
\mathbf{v}(x)=\mathbf{h}_{o}(x) \mathbf{v}(0)+\mathbf{h}_{1}(x) \mathbf{v}^{\prime}(0) \\
\mathbf{h}_{o}(x)=\mathbf{h}^{\prime}(x) \mathbf{M}+\mathbf{h}(x) \mathbf{C}, \quad \mathbf{h}_{1}(x)=\mathbf{h}(x) \mathbf{M} \\
\mathbf{v}(x)=\mathbf{h}(x) \mathbf{c}+\mathbf{h}^{\prime}(x) \mathbf{c}_{2}
\end{gathered}
$$

for constant $2 \times 1$ vectors $\mathbf{c}_{1}$ and $\mathbf{c}_{2}$ [8]. Here $\mathbf{h}(x)$ is the $2 \times$ matrix solution of the initial value problem

$$
\begin{gathered}
\mathbf{A} \mathbf{h}^{\prime \prime}(\mathbf{x})+\mathbf{B} \mathbf{h}^{\prime}(\mathbf{x})+\mathbf{C}(\lambda) \mathbf{h}(\mathbf{x})=\mathbf{0}, \\
\mathbf{h}(0)=\mathbf{0}, \quad \mathbf{A h}^{\prime}(0)=\mathbf{I} .
\end{gathered}
$$

where $\mathbf{0}$ denotes the $2 \times 2$ null matrix and $\mathbf{I}$ the $2 \times 2$ identity matrix. The matrix coefficients being given as in (40).

For any cantilever beam of length $L$, we have the condition $\mathbf{v}(0)=0$ and the bending moment and shear given at $x=L$. From the initial values of $\mathbf{h}(x)$, it follows that $\mathbf{c}_{2}=\mathbf{0}$. Thus we have to determine $\lambda$ so that

$$
\mathbf{v}(x)=\mathbf{h}(\lambda, x) \mathbf{c}
$$

satisfies the boundary condition at the free end, that is

$$
\mathbf{U} \mathbf{c}=\left(\mathbf{F h}(\lambda, \mathbf{L})+\mathbf{Q h}^{\prime}(\lambda, \mathbf{L})\right) \mathbf{c}=\mathbf{0}
$$

From this, it turns out the characteristic equation

$$
\Delta(\lambda)=\operatorname{det}(\mathbf{U})=\mathbf{0} .
$$

We should observe that the modes have the same shape, regardless of the conditions at the free end, but the eigenvalue $\lambda$ differs according to the boundary coefficient matrices $F$ and $Q$. 


\section{$\mathbf{h}(x)$ in closed form}

The above characteristic equations can be determined by computing the fundamental matrix solution $\mathbf{h}(x)$. This later can be given in closed form as [8] [9]

$$
\mathbf{h}(x)=\left(\begin{array}{cc}
a_{1} d(x)+b_{1} d^{\prime \prime}(x) & -a_{1} d^{\prime}(x) \\
a_{1} d^{\prime}(x) & c_{1} d(x)+s_{1} d^{\prime \prime}(x)
\end{array}\right)
$$

where $\quad a_{1}=\kappa G A+\lambda^{2} \rho I, b=-E I, c_{1}=\lambda^{2} \rho A \quad$ and $s_{1}=-\kappa G A$. The scalar solution $d(x)$ is given by

$$
d(x)=\frac{\delta \sinh (\varepsilon x)-\varepsilon \sin (\delta x)}{a b\left(\varepsilon^{2}+\delta^{2}\right) \varepsilon \delta} .
$$

Here

$\varepsilon=\frac{\sqrt{-2 g^{2}+2 \sqrt{g^{4}+4 r^{4}}}}{2}, \quad \delta=\frac{\sqrt{2 g^{2}+2 \sqrt{g^{4}+4 r^{4}}}}{2}$

where

$$
g^{2}=-\frac{(a e+b c) \lambda^{2}}{a b}, r^{4}=\frac{\lambda^{4} c e+\lambda^{2} c a}{a b}
$$

and $a=\kappa G A, b=E I, c=\rho A, e=\rho I$. We should observe that the behaviour of the parameters $\varepsilon$ and $\delta$ as functions of the eigenvalue $\lambda$ can be very complex due to the nonlinear nature of the coefficients of the characteristic polynomial.

It we take a closer look at the roots, we observe that $\delta$ is always a positive number while $\varepsilon$ could be a real or a pure imaginary number $i \varepsilon$. The critical value $\varepsilon=0$ occurs when $\beta^{2}=\frac{E I}{\rho A L^{4}} \lambda^{2}$ equals to the critical value $\beta_{c}=\frac{\kappa G A}{\rho I}$. For $\beta>\beta_{c}$, the solution $d(x)$ becomes pure oscillatory

$$
d(x)=\frac{\delta \sin (\varepsilon x)-\varepsilon \sin (\delta x)}{a b\left(\delta^{2}-\varepsilon^{2}\right) \varepsilon \delta} .
$$

\section{THE GALERKIN APPROXIMATION OF THE MATRIX RESPONSE $h(t, \xi, \mu)$}

The Galerkin method [25], [26] can be used for determining approximate forced vibrations of the cantilever Timoshenko beam model. For this, we introduce the block matrix

$$
\mathbf{V}(x)=\left(\begin{array}{llll}
\mathbf{v}_{1}(x) & \mathbf{v}_{2}(x) & \ldots & \mathbf{v}_{n}(x)
\end{array}\right)
$$

whose columns are the cantilever eigenfunctions that share the normal mode property, that is,

$$
\mathbf{v}_{j}(x)=\left(\begin{array}{c}
w_{j}(x) \\
\psi_{j}(x)
\end{array}\right)=\mathbf{h}\left(x, \beta_{j}\right) \mathbf{c}_{j}
$$

where $\mathbf{c}_{j}$ is obtained by finding a nonzero solution of Eqn. (44). We shall assume that the eigenfunctions of the cantilever AFM microbeam have been normalized with respect to the mass matrix $M$. We now consider the obtention of an approximate forced response

$$
\mathrm{v}(t, x) \doteq \sum_{j=1}^{n} p_{j}(t) \mathrm{v}_{j}(x)=\mathbf{V}(x) \mathbf{P}(t),
$$

of the cantilever Timoshenko model subject to a linearized elastic, damping and inertia forcing localized at $x=L$ as in Eqn. (14)

$$
\mathrm{Mv}_{\mathrm{tt}}+\mathrm{Kv}=\mathrm{F}
$$

where $\mathrm{F}=\left[-\mathrm{Gv}(t, x)-\mathrm{Pv}_{t}(t, x)-\mathrm{Sv}_{t t}(t, x)\right] \delta(x-L)$ with $\mathrm{G}, \mathrm{PS}$ given in Eqn. (14). It will be assumed that the microbeam is subject to initial conditions $\mathrm{v}(0, \mathrm{x})=\mathrm{v}_{\mathrm{o}}(\mathrm{x}), \mathrm{v}_{\mathrm{t}}(0, \mathrm{x})=\mathrm{v}_{1}(\mathrm{x})$.

For determining the time amplitudes $\mathbf{P}^{T}(t)=$ $\left(\begin{array}{llll}p_{1}(t) & p_{2}(t) & \ldots & p_{n}(t)\end{array}\right)$, we substitute Eqn. (49) into Eqn. (52), pre-multiply the resulting matrix differential equation by $\mathbf{V}(\mu)^{T}$ and integrate in order to apply the normal mode property. It turns out the $n$ dimensional system

$$
\ddot{\mathbf{P}}(t)+\Omega^{2} \mathbf{P}(t)=\mathrm{f}
$$

where

$$
\Omega^{2}=\left(\begin{array}{cccc}
\omega_{1}^{2} & 0 & \cdot & 0 \\
0 & \omega_{2}^{2} & \cdot & 0 \\
\cdot & \cdot & \cdot & \cdot \\
0 & 0 & \cdot & \omega_{N}^{2}
\end{array}\right)
$$

is the spectral matrix of the microcantilever with natural frequencies are $\lambda=i \omega$ and $\mathrm{f}(t)=\int_{0}^{L} \mathbf{V}(\mu)^{T} \mathrm{~F}(t, \mu) d \mu$.

The forced response of Eqn. (53) can be written as

$$
\mathbf{P}(t)=\int_{0}^{t} \mathbf{h}(t-v) \mathrm{f}(\mathrm{t}) v
$$

where $\mathbf{h}(t)$ satisfies $\ddot{\mathbf{h}}(t)+\Omega^{2} \mathbf{h}(t)=0, \mathbf{h}(0)=\mathbf{0}, \dot{\mathbf{h}}(0)=\mathbf{I}[8]$. By substituting Eqn. (55) in the approximated forced response $\mathrm{v}(t, x)=\mathbf{V}(x) \mathbf{P}(t)$, we obtain the Galerkin approximation

$$
\mathbf{h}(t, \xi, \mu) \doteq \mathbf{V}(x) \mathbf{h}(t) \mathbf{V}^{T}(\xi)
$$

Due to the decoupled character of the above equations, we have that the fundamental matrix response $h(t)$ can be written as

$\mathbf{h}(t)=\frac{\sin (\Omega t)}{\Omega}=\left(\begin{array}{cccc}\frac{\sin \left(\omega_{1} t\right)}{\omega_{1}} & 0 & \ldots & 0 \\ 0 & \frac{\sin \left(\omega_{2} t\right)}{\omega_{2}} & \ldots & 0 \\ \cdot & \cdot & \cdot & \cdot \\ 0 & 0 & \cdot & \frac{\sin \left(\omega_{N} t\right)}{\omega_{N}}\end{array}\right)$

From (56) and (57), we have the frequency matrix approximation $\mathbf{H}(s, \xi, \mu) \doteq \mathbf{V}(\xi)\left(s^{2} \mathbf{I}+\Omega^{2}\right)^{-1} \mathbf{V}^{T}(\mu)$.

Simulations with harmonic and a rectangular pulse forcing were performed at the free end of the beam and subject to a tip-sample interaction modeled as vertical and lateral dashpots [4]. It is observed that free oscillations that appear with the modes of a cantilever beam are somehow damped when subject to tip-sample conditions at the end. Also, the rotation, that will correspond to slope in the Euler-Bernoulli model, has significant influence and it should be attributed to the inclusion of the rotatory inertia and shear deformation in the Timoshenko model. 


\section{CONCLUSIONS}

This paper has formulated in matrix form the basic dynamic modes of operation of atomic force microscopy as a uniform Timoshenko beam of finite length subject to tip-sample interactions and external excitations. It has been made extensive use of the distributed matrix impulse response for predicting forced responses. The mode shapes and characteristic equations have been obtained by solving a second-order damped differential equation with matrix coefficients. It was shown that there is a critical cut-off frequency above which the behaviour completely oscillatory. The Galerkin approximation was employed with cantilever eigenfunctions for approximating the impulse matrix response. Simulations included harmonic excitations and pulse with tip-sample interaction devices. The procedure employed with normal modal analysis can be also used with nonclassical modes in a wide range of practical situations. Also, with composite beams that include piezoelectric materials.

\section{ACKNOWLEDGMENTS}

This work was partially done when the first author was visiting the Department of Mathematics at the UFSM-Santa Maria and the Laboratory of Modeling and Control of the DEMAC-IGCE at UNESP- Rio Claro.

\section{REFERENCES}

1. G. Binnig, C. F. Quate, and C. Gerber, Physics Review Letters 56, 930-933 (1986).

2. Y. Song, and B. Bhushan, Journal of Physics: Condensed Matter 20, 225012-225041 (2008).

3. H. L. Lee, S. S. Chu, and W. J. Wang, Current Applied Physics 10, 570-573 (2010).

4. J. Hsu, H. L. Lee, and W. Chang, Nanotechnologye 18, 28503-28508 (2007)

5. J. A. Turner, S. Hirsekorn, U. Rabe, and W. Arnold, Journal of Applied Physics 82, 966-979 (1997).

6. K. Wolf, and O. Gottlieb, Journal of Applied Physics 91, 4701-4709 (2002).

7. S. I. Lee, S. W. Howell, A. Raman, and R. Reifenberger, Physical Review B 66, 115409 (2002).

8. J. R. Claeyssen, G. Canahualpa, and C. Jung, Applied Numerical Mathematics 30, 65-78 (1999).

9. J. Claeyssen, and S. Costa, Journal of Sound and Vibration 296, 1053-1058 (2006).

10. J. Ginsberg, Mechanical and Structural Vibrations, John Wiley, 2001.

11. T.C.Huang, Journal of Applied Mechanics 28, 579-584 (1961)

12. H. Hölscher, U. Schwarz, and R. Wiesendanger, Applied Surface Science 140, 344-351 (1999).

13. T. Uchihashi, M. Ohta, Y. Sugawara, Y. Yanase, T. Sigematsu, M. Suzuki, and S. Morita, Journal of Vacuum Science and Technology B 15, 1543-1546 (1997).

14. U. Rabe, E. Kester, and W. Arnold, Surface and Interface Analysis 27, 386-391 (1999).
15. J. Yazdani, S. Yazdani, and S. Bigham, Digest Journal of Nanomaterials and Biostructures 3, 277-285 (2008).

16. M. Sitti, Automatic Control Conference (ACC) pp. 1-16 (2003)

17. H. Butt, B. Cappella, and M. Kappl, Surface Science Reports 59, 1-152 (2005)

18. S. Eslami, and N. Jalili, Ultramicroscopy 117, 31-45 (2012).

19. W. Chang, T. Fang, and C. Weng, Nanotechnology 15, 427-430 (2004)

20. T. L. Horng, Applied Surface Science 256, 311-317 (2009).

21. W. L.Wang, and S. Hu, Applied Physics Letters 87, $183506(2005)$

22. S. Rützel, S. I. Lee, and A. Raman, Proceedings of the Royal Society of London A 459, 1925-1948 (2003).

23. A. Yavari, S. Sarkani, and J. N. Reddy, International Journal of Solids and Structures 38, 8389-8406 (2001).

24. J. R. Claeyssen, Dincon 2010 pp. 1-12 (2010).

25. S. Kelly, Advanced Vibration Analysis, CRCPress, 2007.

26. S. Rao, Vibration of Continuous Systems, John Wiley, 2007.
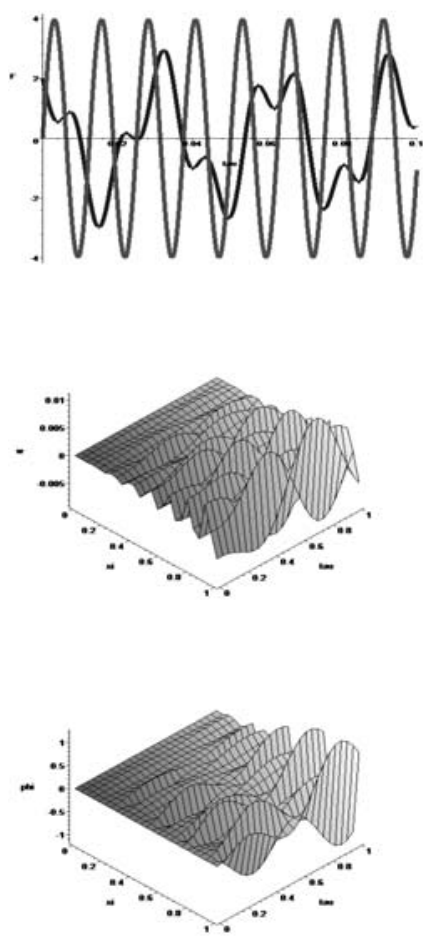

FIGURE 4. Coupled harmonic input $\mathbf{F}=$ $\operatorname{col}[4 \sin (500 t),-\sin (530 t)+2 \cos (200 t)]$ with corresponding displacement $w(t, \xi)$ and rotation $\phi(t, \xi)$ outputs. 


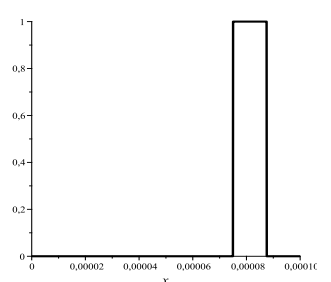

(A)

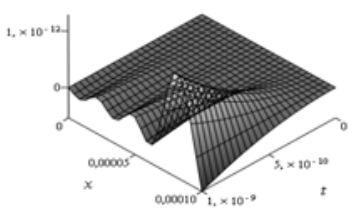

(B)

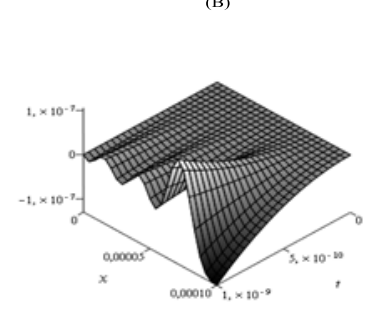

(C)

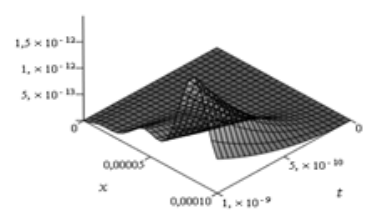

(D)

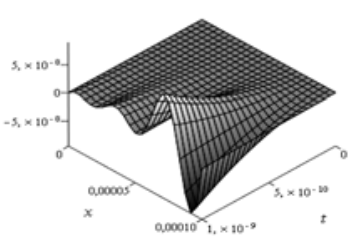

FIGURE 5. Displacement $w(t, \xi)$ and rotation $\phi(t, \xi)$ outputs for clamped-free beam (A),(B) and clamped-free with dashpot devices (C),(D) due to a modulated input of a spatial pulse $\mathbf{F}=\left(\cos \omega_{f} t\right)[$ Heaviside $(x-3 L / 4)-$ Heaviside $(x-$ $7 L / 8)]$.
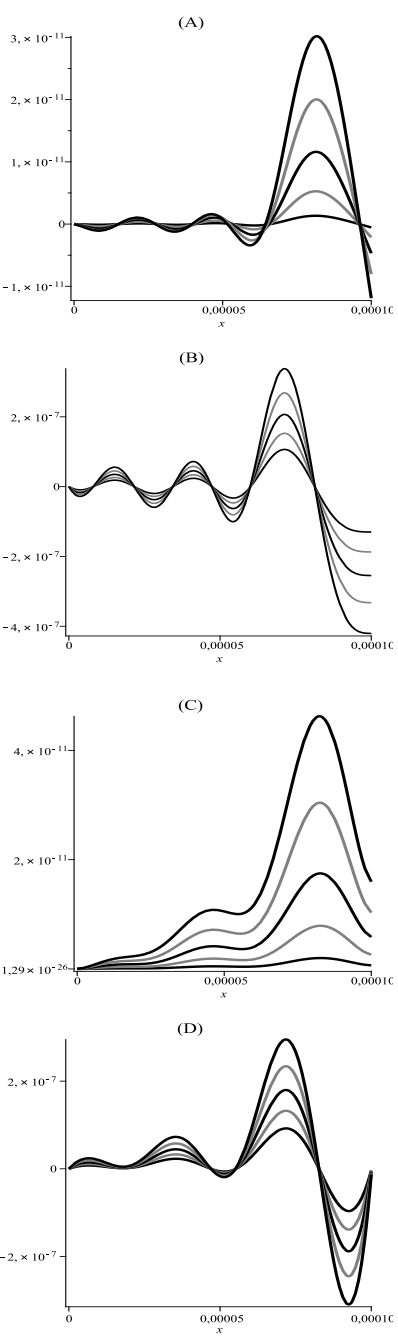

FIGURE 6. Corresponding displacements $w(t, \xi)$ and rotation $\phi(t, \xi)$ for several times $t$. 
Copyright of AIP Conference Proceedings is the property of American Institute of Physics and its content may not be copied or emailed to multiple sites or posted to a listserv without the copyright holder's express written permission. However, users may print, download, or email articles for individual use. 